\title{
Applications of Codense and Compatible Ideals
}

\author{
Shyamapada Modak ${ }^{1}$, Sukalyan Mistry ${ }^{2}$ \\ ${ }_{1,2}$ Department of Mathematics University of Gour Banga Malda 732 103, W.B, India
}

\begin{abstract}
In this paper, we will discuss the further properties of some new types of set which have been defined with the help of ideal. We also characterize these sets, generalized continuities and * extremally disconnectedness in ideal topological spaces.

Keywords: $t$-I-set, RI - open set, weakly I-locally closed set, $\mathcal{A}_{I R}$-set.
\end{abstract}

\section{Introduction}

The subject of ideals in topological spaces has been studied by Kuratowski [22] and Vaidyanathaswamy [35]. An ideal on a topological space $(X, \tau)$ as a non-empty collection $I$ of subsets of $X$ satisfying the following two conditions:

(1) If $A \in I$ and $B \subset A$, then $B \in I$;

(2) If $A \in I$ and $B \in I$, then $A \cup B \in I$.

An ideal topological space [7] is a topological space $(X, \tau)$ with an ideal $I$ on $X$, and is denoted by $(X, \tau, I)$. An ideal in an ideal topological space $(X, \tau, I)$ is called a codense ideal [8] if $I \cap \tau=\{\varnothing\}$. Although Newcom [31], Jankovic and Hamlett [18] have used this as $\tau$-boundary where as Dontchev [6] calls such spaces as Hayasi-Samuel spaces. In fact such ideals play very important role in the study of ideal topological spaces. Some remarkable results have been considered in $[26,27,18,15,34,25,30,29,28,13]$ using this ideal. Another important ideal is compatible ideal which had been introduced by Jankovic and Hamlett [19]. Mathematicians like Rose, Hamlett and Jankovic in [19, 34] and Modak and Bandyopadhyay in [26, 27, 5] have studied it extensively. An ideal $I$ is said to be compatible with $\tau$, denoted by $I \sim \tau$, for each $A \subset X$ and for each $a \in A$ there exists a neighbourhood $U$ of $a$ such that $U \cap A \in I$ then $A \in I$.

For a subset $A \subset X$, the set $A^{*}(I)=\{x \in X: U \cap A \notin I\}$ for every $U \in \tau$ with $x \in U$, is called the local function of $A$ with respect to $I$ and $\tau$ [22]. We simply write $A^{*}$ instead of $A^{*}(I)$ in case there is no chance of confusion. It is well known that $C l^{*}(A)=A \cup A^{*}$ defines a kuratowski closure operator for $\tau^{*}(I)$ and one of its base is $\{U-A: U \in \tau, A \in I\}$. It is interesting that $\tau^{*}(I)=\{U-A: U \in \tau, A \in I\}$ when $\tau \sim I$ [19]. In this ideal topological space a set $A$ is called *-dense [8] if $C l^{*}(A)=X$, and the set $A$ is called $I-$ open if $A \subset$ Int $\left(A^{*}\right)$ Again in ideal topological space, Modak and Bandyopadhyay [26, 27] have proved two remarkable results: $C l(O)=O^{*}$, for the open set $O$ if and only if $I \cap \tau=\{\varnothing\}$; and for any nonempty $G \in \tau^{*}(I), C l^{*}(G)=$ $C l(G)$, when $I$ is codense and $I \sim \tau$.

The study of generalized sets in the topological space and in the ideal topological space are the important part in the study of topological spaces.

Let space $(X, \tau)$ be a topological space and $A \subset X . A$ is said to be preopen [24] (resp. $\alpha$ - open [32], semi open [23], semi - preopen [3], b - open [2]) if $A \subset \operatorname{Int}(\operatorname{Cl}(A))(\operatorname{resp} . A \subset \operatorname{Int}(\operatorname{Cl}(\operatorname{Int}(A))), A \subset$

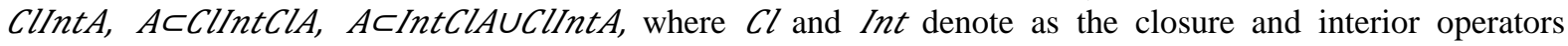
respectively. The collection of all preopen (resp. $\alpha$ - open, semi - open, semi - preopen, $b$ - open) sets in $(X, \tau)$ is denoted by $P O(X, \tau)$ (resp. $\alpha O(X, \tau), S O(X, \tau), S P O(X, \tau), B O(X, \tau))$.

$A$ subset $A$ of an ideal topological space $(X, \tau, I)$ is said to be pre - $I$-open [6] (resp. semi - $I$ - open [17], $\alpha$ - $I$ - open [17], $\beta$ - I - open [17], $b$ - I - open [14], strongly $\beta$ - I - open [16], $t$ - $I$ - set [17], semi - $I$ regular [21], $A B_{I}$ - set [21], weakly $I$ - locally closed ([20] [21]) if $A \subset \operatorname{Int}\left(C l^{*}(A)\right)\left(r e s p . A \subset C l^{*}(\operatorname{Int}(A)), A \subset \operatorname{Int}\left(C l^{*}(\operatorname{Int}(A))\right), A \subset C l\left(\operatorname{Int}\left(C l^{*}(A)\right)\right)\right.$,

$A \subset \operatorname{Int}\left(C l^{*}(A)\right) \cup C l^{*}(\operatorname{Int}(A)), A \subset C l^{*}\left(\operatorname{Int}\left(C l^{*}(A)\right)\right), \operatorname{Int}(A)=\operatorname{Int}\left(C l^{*}(A)\right), A$ is a $t$ - set and semi $I$ - open, $A=U \cap V$ where $U$ is open and $V$ is semi $-I-\operatorname{regular}, A=U \cap K$ where $U$ is open and $K$ is * closed). The collection of all pre - $I$ - open (resp. semi - $I$ - open, $\alpha-I$ - open, $b-I$ - open, Weakly $I$ - locally closed, strongly $\beta-I$ - open) sets is denoted by $\operatorname{PIO}(X)$ (resp. $\operatorname{SIO}(X), \alpha I O(X), B I O(X), W I L C(X)$, $s \beta I O(X))$.

A space $(X, \tau)$ is called extremally disconnected if $\operatorname{Cl}(O)$ is open for every $O \in \tau$, and the space called submaximal space if every dense set is open.

An ideal topological space $(X, \tau, I)$ is said to be $*$ - extremally disconnected [10] if the $C l^{*}(O)$ is open, for every open set $O$, and it is called $I$ - submaximal [11] if every $*$ - dense subset of $X$ is open. 


\section{II. *-Extremally Disconnected Spaces}

Theorem 2.1. For an ideal topological space $(X, \tau, I)$ following hold:

(1) Every semi - open set is $b-I$ - open, when I is codense.

(2) Every preopen set of $\left(X, \tau^{*}(I)\right)$ is $b-I$ - open, when I is codense.

(3) Every I - open set is $b-I$ - open, when I is codense.

(4) Every $b-I-$ open set is semi - preopen (or $\beta$ - open) set of $\left(X, \tau^{*}(I)\right)$, when $I \sim \tau$ and I is condense. Proof. The proof is straightforward from Proposition 1 of [14].

If $I$ is a condense ideal in the ideal topological space $(X, \tau, I)$, then the concept of * maximally disconnected and the extremally disconnected are coincident.

Let $(X, \tau, I)$ be the extremally disconnected space. Then for $V \in \tau, C l(V)$ is open. From [26, 27], $V^{*}=$ $C l(V)$ is open. This implies that $V \cup V^{*}$ is open, and hence $C l^{*}(V)$ is open. So the space $(X, \tau, I)$ is the * extremally disconnected. Reciprocally suppose that the space $(X, \tau, I)$ is $*$ - extremally disconnected. Then for $V \in \tau, C l^{*}(V)$ is open. From [26, 27], $V^{*}=C l(V)$, since $I$ is codense. So $C l^{*}(V)=V \cup V^{*}=C l(V)$, and it is open.

Theorem 2.2. For an ideal topological space $(X, \tau, I)$ where I is codense, then the following properties are equivalent:

(1) $X$ is extremally disconnected,

(2) $C l^{*}(\operatorname{Int}(V)) \subset \operatorname{Int}\left(C l^{*}(V)\right)$ for every subset $V$ of $X$.

Proof. Proof is obvious from [10].

Theorem 8 of [10] can be restated by the following, using extremally disconnectedness.

Theorem 2.3. Let $(X, \tau, I)$ be an extremally disconnected ideal topological space, where I is codense. Then for $A \subset X$, the following properties are equivalent:

(1) $A$ is an open set,

(2) $A$ is $\alpha$ - I - open and weakly I - local closed,

(3) A is pre - I - open and weakly I - local closed,

(4) A is semi - I - open and weakly I - local closed,

(5) $A$ is $b-I$ - open and weakly I-local closed.

Theorem 2.4. Let $(X, \tau, I)$ be an extremally disconnected ideal space, where I is codense. Then for $A \subset X$, the following properties are equivalent:

(1) $A$ is an open set,

(2) A is $\alpha-I$ - open and a locally closed set,

(3) A is pre - I - open and a locally closed,

(4) A is semi - I - open and a locally closed set,

(5) A is $b-I$ - open and a locally closed set.

Proof. The proof of this theorem is obvious from Theorem 9 of [10] and [27].

Now we shall discuss about decompositions of continuous functions. For this we define following:

Definition 2.5. A function $f:(X, \tau, I) \rightarrow(Y, \sigma)$ is $\alpha$ - I - continuous [17, 18] ( resp. pre - $I$ - continuous [7], semi - $I$ - continuous [17], $b-I$ - continuous [14], $W_{I} L C$ - continuous [20], $L C$ - continuous [12] ) if $f^{-1}(V)$ is $\alpha$ - $I$ - open ( resp. pre - $I$ - open, semi - $I$ - open, $b$ - I - open, weakly $I$ - locally closed, locally closed ) for each open set $V$ in $Y$.

If $I$ is codense, then every semi-open set is a $b-I$ - open set, and every preopen set of $\tau^{*}(I)$ is a $b-I-$ open set. Therefore every semicontinuous function is a $b-I$ - continuous function, and every precontinuous function on $\left(X, \tau^{*}(I)\right)$ is always a $b-I$ - continuous function.

We can replaced the term * - extremally disconnected by extremally disconnected in Theorem 26 of [10].

Theorem 2.6. For a function $f:(X, \tau, I) \rightarrow(Y, \sigma)$, where $(X, \tau, I)$ is an extremally disconnected ideal space and $I$ is codense, the following properties are equivalent:

(1) $f$ is continuous,

(2) $f$ is $\alpha-I$ - continuous and $W_{I} L C$ - continuous,

(3) $f$ is pre - I - continuous and $W_{I} L C$ - continuous,

(4) $f$ is semi - I - continuous and $W_{I} L C$ - continuous,

(5) $f$ is $b-I$ - continuous and $W_{I} L C$ - continuous.

According to the intimation of codense ideal we can further discuss of Theorem 27 of [10]. 
Theorem 2.7. Let $f:(X, \tau, I) \rightarrow(Y, \sigma)$ be a function, where $(X, \tau, I)$ is an extremally disconnected and I is codense. Then following properties are equivalent:

(1) $f$ is continuous,

(2) $f$ is $\alpha-I$ - continuous and LC - continuous,

(3) $f$ is pre - I - continuous and LC - continuous,

(4) $f$ is semi - I - continuous and LC - continuous,

(5) $f$ is $b-I$ - continuous and LC - continuous.

We rewrite the Lemma 15 of [11] using codense ideal.

Theorem 2.8. For an ideal space $(X, \tau, I)$ where I is codense, the following properties are equivalent:

(1) $X$ is extremally disconnected,

(2) Every semi - I - open set is pre - I - open,

(3) The * - closure of every strongly $\beta-I$ - open subset of $X$ is open,

(4) Every strongly $\beta$ - I - open set is pre - I - open.

We are ending this section with the following Remark for importance of codense ideal.

Remark 2.9. Let $(X, \tau, I)$ be an ideal topological space with $I \cap \tau=\{\varnothing\}$. Then $X$ is $*$-extremally disconnected if and only if it is extremally disconnected.

Proof. Proof is obvious from Theorem 20 of [10].

\section{I-Submaximal Spaces}

In previous section, we have interrelated the $*$ - extremally disconnected spaces and the extremally disconnected spaces. In this section we try to interrelate the submaximal spaces and the $I$ - submaximal spaces in presence of restriction on ideal. Although Modak in [26, 27] have proved following:

Theorem 3.1. If $(X, \tau)$ is extremally disconnected and submaximal then $\left(X, \tau^{*}(I)\right)$ is extremally disconnected and submaximal, where $I \sim \tau$ and $I \cap \tau=\{\varnothing\}$.

the space $(X, \tau, I)$

He has also given an example for encounter of submaximality of $\tau^{*}(I)$ implies submaximality of $\tau$ in

Here we further discuss the properties of $I$ - submaximality.

Theorem 3.2. For a subset $A$ of an ideal space $(X, \tau, I)$ where $I$ is codense, the following properties are equivalent:

(1) $A \in P O\left(X, \tau^{*}(I)\right)$,

(2) $A=G \cap B$, where $G$ is open and $B$ is * - dense.

Theorem 3.3. Let $(X, \tau, I)$ be an ideal space, where I is codense and $A \subset X$. Then $A$ is $\alpha-I-$ open if and only if it is semi-open and pre - I - open in $\left(X, \tau^{*}(I)\right)$.

Theorem 3.4. For an ideal topological space $(X, \tau, I)$ where I is codense, the following properties are equivalent:

(1) $X$ is I - submaximal,

(2) Every pre - open set in $\left(X, \tau^{*}(I)\right)$ is open,

(3) Every pre - open set in $\left(X, \tau^{*}(I)\right)$ is semi-open and every $\alpha$-open set is open.

Proof. We know from Theorem 3 of [11]:

$X$ is $I$ - submaximal $\Rightarrow$ Every pre - $I$ - open set is open $\Rightarrow$ Every pre - $I$ - open set is semi - $I$-open and every $\alpha$ $I$ - open set is open.

Then $X$ is $I$ - submaximal $\Rightarrow$ Every pre - open set in $\left(X, \tau^{*}(I)\right)$ is open $\Rightarrow$ Every pre - open set in $\left(X, \tau^{*}(I)\right)$ is semi-open and every $\alpha$ - open set is open (since $I$ is condense).

Proof of the reverse part is similar.

Theorem 3.5. For a subset $A$ of an I-submaximal ideal space $(X, \tau, I)$ where I is codense, the following are equivalent:

(1) A is semi-open,

(2) A is strongly $\beta$ - I - open.

Theorem 3.6. For an ideal space $(X, \tau, I)$ where I is codense, the following are equivalent:

(1) $X$ is I - submaximal,

(2) Every pre - open set in $\left(X, \tau^{*}(I)\right)$ is an $A B_{I}-$ set,

(3) Every * - dense set is an $A B_{I}$ - set.

Proof. We know from Theorem 10 of [11],

$X$ is $I$ - submaximal $\Leftrightarrow$ every pre $-I$ - open set is an $A B_{I}$ - set $\Leftrightarrow$ Every $*$ - dense set is an $A B_{I}$ - set.

Therefore we get, 
$X$ is $I$ - submaximal $\Leftrightarrow$ every pre-open set in $\left(X, \tau^{*}(I)\right)$ is an $A B_{I}$ - set $\Leftrightarrow$ Every $*$-dense set is an $A B_{I}$ - set.

Theorem 3.7. For an ideal space $(X, \tau, I)$ where $I$ is codense, the following are equivalent:

(1) $X$ is I-submaximal and extremally disconnected,

(2) Any subset of $X$ is strongly $\beta-I-$ open if and only if it is open.

Theorem 3.8. For an ideal space $(X, \tau, I)$, where $I$ is codense, if $X$ is I-submaximal and extremally disconnected, the following are equivalent for a subset $A \subset X$ :

(1) A is strongly $\beta-I-$ open,

(2) A is semi - I-open,

(3) A is pre $-I-$ open,

(4) A is $\alpha-I-o p e n$,

(5) A is open.

Proof. Proof is obvious from Corollary 17 of [11].

Theorem 3.9. Every $A B_{I}$ - set is semi-open in an ideal topological space $(X, \tau, I)$, where I is codense.

Proof. Proof is obvious from Lemma 18 of [11].

Theorem 3.10. For an ideal space $(X, \tau, I)$, where $I$ is codense, if $X$ is $I$ - submaximal and extremally disconnected, the following properties are equivalent for a subset $A \subset X$ :

(1) A is semi-I-open,

(2) $A$ is an $A B_{I}-$ set.

Proof. Proof is similar with the Theorem 19 of [11].

\section{RI-Open Sets}

At the starting of this section we shall discuss the following sets which are already in literature.

Let $(X, \tau, I)$ be an ideal space. A subset $A$ of $X$ is said to be a semipre ${ }^{*}-I$ - closed [33] (resp. RI - open set [36]) if $\operatorname{Int}\left(C l^{*}(\operatorname{Int}(A))\right) \subset A\left(\operatorname{resp} . A=\operatorname{Int}\left(C l^{*}(A)\right)\right)$. We will denote the family of all $R I$ - open sets by $R I O(X)$.

A subset $A$ of an ideal space $(X, \tau, I)$ is said to be a $\mathcal{A}_{1 I}$ [33] ( resp. $\mathcal{B}_{1 I}$ [4]) if $A=U \cap V$ where $\mathrm{U}$ is open ( resp. $\alpha-I$ - open) and $C l^{*}(\operatorname{Int}(V))=X$. We will denote the family of all $\mathcal{B}_{1 I}$-sets (resp. $\mathcal{A}_{1 I}$ - sets) by $\mathcal{B}_{1 I}(X)\left(\right.$ resp. $\left.\mathcal{A}_{1 I}(X)\right)$.

A subset $A$ of an ideal space $(X, \tau, I)$ is said to be a $\mathcal{B}_{I}$ - set [17] (resp. $\mathcal{C}_{I}$ - set [17]) if $A=U \cap V$ where $U$ is open and $V$ is a $t-I-$ set (resp. semipre ${ }^{*}-I-$ closed set). We will denote the family of all $\mathcal{B}_{I}$ - set (resp. $\mathcal{C}_{I}$ - set) by $\mathcal{B}_{I}(X)\left(\right.$ resp. $\mathcal{C}_{I}(X)$ ).

A subset $A$ of an ideal space $(X, \tau, I)$ is said to be a $\mathcal{A}_{2 I}$ - set [4] ( resp. $\mathcal{B}_{2 I}$ - set [4] ( $\alpha_{1} M_{2}$ - set [1] ) if $A=U \cap V$ where $U$ is open ( resp. $\alpha-I$ - open ) and $C l^{*}(V)=X$. We will denote the family of all $\mathcal{A}_{2 I}$ sets (resp. $\mathcal{B}_{2 I}$ - sets) by $\mathcal{A}_{2 I}(X)$ (resp. $\left.\mathcal{B}_{2 I}(X)\right)$. Clearly $\mathcal{A}_{2 I}(X) \subset \mathcal{B}_{2 I}(X)$.

A subset $A$ of an ideal space $(X, \tau, I)$ is said to be an $\alpha_{I} N_{5}$ - set [1] if $A=U \cap V$ where $U$ is $\alpha-I$ open and $V$ is $*$ - closed. We will denote the family of all $\alpha_{I} N_{5}$ - sets of an ideal space $(X, \tau, I)$ by $\alpha_{I} N_{5}(X)$.

A subset $A$ of an ideal space $(X, \tau, I)$ is said to be $I R$ - closed set [36] if $A=C l^{*}(\operatorname{Int}(A))$.

A subset $A$ of an ideal space $(X, \tau, I)$ is said to be an $\alpha \mathcal{A}_{I}$ - set [4] (resp. $\mathcal{A}_{I R}$ - set [1]) if $A=U \cap V$ where $U$ is an $\alpha-I-$ open (resp. open) set and $V$ is an $I R$ - closed. $\mathcal{A}_{I R}$ - sets are called as $\mathcal{A}_{I}$ - sets in [4].

A subset $A$ of an ideal space $(X, \tau, I)$ is said to be a $\mathcal{A}_{3 I}$ - set [33] if $A=U \cap V$ where $U$ is open and $C l^{*}(\operatorname{Int}(V)) \subset V$. We will denote the family of all $\mathcal{A}_{3 I}$ - sets by $\mathcal{A}_{3 I}(X)$

Now we shall discuss some characterizations of the above sets.

Theorem 4.1. Let $(X, \tau, I)$ be an ideal space $(X, \tau, I)$, where I is codense and $A \subset X$. Then

$\mathcal{B}_{1 I}(X)=\alpha I O(X)=\mathcal{A}_{1 I}=\tau^{\alpha}$.

Proof. Proof is similar with the Theorem 2.8 of [33] and the condition $O^{*}=\mathrm{Cl}(O)$ for open set $O$.

Theorem 4.2. Let $(X, \tau, I)$ be an ideal space where I is codense. Then following are equivalent:

(1) A is pre -I - open,

(2) A is pre - open in $\left(X, \tau^{*}(I)\right)$,

(3) There exists an RI- open set $G$ such that $A \subset G$ and $C l^{*}(G)=C l^{*}(A)$,

(4) $A=G \cap D$ where $G$ is $R I-$ open and $D$ is * - dense,

(5) $A=G \cap D$ where $G$ is open and $D$ is * - dense.

Proof. Proof is obvious from the Theorem 2.9 of [33] and [26, 27].

Theorem 4.3. Let $(X, \tau, I)$ be an ideal space, where $I$ is codense. Then $\mathcal{A}_{2 I}(X)=\operatorname{PIO}(X)=\mathcal{B}_{2 I}(X)=$ $P O\left(X, \tau^{*}(I)\right)$.

Proof. Proof is obvious from the [26, 27]. 
Theorem 4.4. Let $(X, \tau, I)$ be an ideal space, where $I$ is codense and $A \subset X$. Then $A$ is $\alpha_{I} N_{5}-$ set if and only if $A=U \cap C l^{*}(A)$ for some $U \in \tau^{\alpha}$.

Proof. Proof is obvious from Theorem 2.13 of [33] and [26, 27].

Theorem 4.5. Let $(X, \tau, I)$ be an ideal space where I is codense. Then $\alpha \mathcal{A}_{I}(X)=\operatorname{siO}(X) \cap \alpha_{I} N_{5}(X)=$ $\operatorname{SIO}(X)=\operatorname{SO}(X)$.

Proof. Proof is obvious from the fact that $I \cap \tau=\{\varnothing\}$ if and only if $O^{*}=\operatorname{Cl}(O)$ for any open set $O$.

Theorem 4.6. Let $(X, \tau, I)$ be an ideal space and $A \subset X$, where I is codense. Then following are equivalent:

(1) A is $\alpha-$ open.

(2) A is $\alpha-I-$ open.

(3) A is pre - I - open and semi - I - open.

(4) $A$ is a $\mathcal{B}_{2 I}-$ set and $\alpha \mathcal{A}_{I}-$ sets.

Proof. Proof is obvious from Proposition 1.1, Theorem 2.3 of [4], Corollary 2.15 of [33] and [26, 27].

Theorem 4.7. Let $(X, \tau, I)$ be an ideal space, where $I$ is codense and $A \subset X$. Then the following are equivalent:

(1) A is open.

(2) $A$ is $\alpha-I-$ open and $\mathcal{A}_{I R}-$ set.

(3) $A$ is $\alpha$ - open and $\mathcal{A}_{I R}-$ set.

(4) A is pre-open set in $\left(X, \tau^{*}(I)\right)$ and $\mathcal{A}_{I R}$ - set.

(5) A is $\alpha-I-$ open and weakly I - locally closed.

(6) $A$ is $\alpha-$ open and $\mathcal{B}_{I}-$ set.

(7) $A$ is $\alpha-I-$ open and $\mathcal{C}_{I}-$ set.

Proof. Proof is obvious from Corollary 2.16 of [33] and [26, 27].

Theorem 4.8. Let $(X, \tau, I)$ be an ideal space, where $I$ is codense and $A \subset X$. Then the following are equivalent.

(1) $A \in \mathcal{A}_{I R}(X)$.

(2) $A \in S O(X) \cap \mathcal{A}_{3 I}(X)$.

(3) $A \in \alpha \mathcal{A}_{I}(X) \cap \mathcal{A}_{3 I}(X)$.

(4) $A \in S \beta I O(X) \cap \alpha_{I} N_{5}(X) \cap \mathcal{A}_{3 I}(X)$.

(5) $A \in s \beta I O(X) \cap \operatorname{WILC}(X)$.

Proof. Proof is obvious from Corollary 2.20 of [33] and [26, 27].

\section{References}

[1] A. Acikgoz and S. Yuksel, Some new sets and decompositions of $A_{I-R}$-continuity, $\alpha$-I-continuity, Continuity via Idealization, Acta Math. Hungar., 114(1-2)(2007), 79 - 89.

[2] D. Andrijevic, On b-open sets, MATHEMAT, 48 (1996), 59 - 64

[3] D. Andrijevic, On the topology generated by preopen sets, Mathemathhkh Bechhk, 39 (1987), $367-376$.

[4] G. Aslim, A. C. Guler and T. Noiri, On decompositions of continuity and some weaker forms of continuity via idealization, Acta Math. Hungar., 109(3) (2005), 183 - 190

[5] C. Bandyopadhyay and S. Modak, A new topology via $\psi$-operator, Proc. Nat. Aca. Sci.India, 76(A), IV(2006), 317 - 320.

[6] J. Dontehev, On pre-I-open sets and a decomposition of I-continuity, Banyan Math J., 2(1996).

[7] J. Dontehev, Idealization of Ganster-Reilly decomposition theorems, arXIV: Math. Gn/9901017VI, 5 Jan 1999

[8] J. Dontehev, M. Ganster and D. Rose, Ideal resolvability, Topology Appl. 64(3) (1999), 1 - 16.

[9] E. Ekici, On pre-I-open sets, semi-I-open sets and b-I-open sets in ideal topological spaces, Acta Univ. Apulen. 30 (2012), 293 303.

[10] E. Ekici and T. Noiri, * - extremally disconnected ideal topological spaces, Acta Math. Hun- gar., 122(1-2) (2009), 47 - 54.

[11] E. Ekici and T. Noiri, Properties of $I$-submaximal ideal topological spaces, Filomat 24:4 (2010), 87 - 94.

[12] M. Ganster and I. L. Reilly, Locally closed LC-continuous functions, Int. J. Math. Math. Sci. $12(3)$ (1989), 417 - 424.

[13] B. Garai, C. Bandyopadhyay and S. Modak, Topological properties via Ideals, Indian Jour- nal of Mathematics, 48(3) (2006), 347 357.

[14] A. C. Guler and G. Aslim, b - I - open sets and decomposition of continuity via idealization, Proc. Inst. Math. Mech. Natl. Acad. Sci. Azerb., 22 (2005), $27-32$.

[15] T. R. Hamlett and D. Jankovic, Ideals in topological spaces and the set operator $\psi$, Bol-lettino U. M. I., (7) 4-B (1990), 863 - 874

[16] E. Hatir, A. Keskin and T. Noiri, On a new decomposition of continuity via idealization, JP Jour Geometry and Topology, 3(1)(2003), $53-64$.

[17] E. Hatir and T. Noiri, On decomposition of continuity via Idealization, Acta Math. Hungar., 96(4) (2002), 341 - 349.

[18] D. Jankovic and T. R. Hamlett, New topologies from old via ideals, Amer. Math. Monthly, 97(1990), 295 - 310.

[19] D. Jankovic, T. R. Hamlett, Compatible extensions of ideals, Bollettino U. M. I., (7) 6-B(1992), $453-465$.

[20] A. Keskin, T. Noiri and S. Yuksel, Decomposition of I-continuity and continuity, Commun. Fac. Sci. Univ. Ankara Series AI, 53 (2004), $67-75$.

[21] A. Keskin and S. Yuksel, On semi-I-regular sets, $A B_{I}$-sets and decompositions of continuity, $R_{I} C$-continuity, $A_{I}$-continuity, Acta Math. Hungar., 113 (3) (2006), 227-241.

[22] K. Kuratowski, Topology, Vol. I, Academic Press, New York, 1966.

[23] N. Levine, Semi-open sets and semi-continuoty in topological spaces, Amer. Math. Monthly 70 (1963), 36 - 41.

[24] A. S. Mashhour, M. E. Abd El-Monsef and S. N. El-Deep, On precontinuous and week precontinuous mappings, Proc. Math. Phys. Soc. Egypt, 53 (1982), 47 - 53.

[25] S. Modak, Some new topologies on ideal topological spaces, Proc. Natl. Acda. Sci. India, 82 (3) (2012), 233 - 243. 
[26] S. Modak and C. Bandyopadhyay, * - topology and generalized open sets, Soochow J. Math. 32 (2006), 201 - 210

[27] S. Modak and C. Bandyopadhyay, A note on $\psi$-operator, Bull. Malays. Math. Sci. Soc., 30 (1) (2007), 43 - 48.

[28] S. Modak and Md. M. Islam, New form of Njastad's $\alpha$-set and Levines's semi-open set, Journal of the Chungcheong Mathematical Society, 30 (2) (2017), 165 - 175

[29] S. Modak and Md. M. Islam, More on $\alpha$-topological spaces, Commun. Fac. Sci. Univ. Ank. Series A1, 66 (2) (2017), 323 - 331.

[30] S. Modak and T. Noiri, Connectedness of ideal topological spaces, Filomat, 29 (4) (2015), 661 - 665.

[31] R. L. Newcomb, Topologies which are compact modulo an ideal, Ph. D. Dissertation, Univ. of Cal. at Santa Barbara, 1967.

[32] O. Njastad, On some classes of nearly open sets, Pacific J. Math., 15 (1965), 961 - 970.

[33] V. Renukadevi, On subsets of ideal topological spaces, CUBO Math. J., 12(2) 2010, 43 -52.

[34] D. A. Rose and T. R. Hamlett, Ideally equivalent topologies and semi-topological properties, Math. Chron. 20(1991), 149 - 165.

[35] R. Vaidyanathaswamy, Set Topology, Chelsea Publishing Company, 1960.

[36] S. Yukesl, A. Acikgoz and T. Noiri, On $\delta$-I-continuous functions, Turk. J. Math., 29(2005), 39 - 51.

Shyamapada Modak. "Applications of Codense and Compatible Ideals." IOSR Journal of Mathematics (IOSR-JM) 13.3 (2017): 4-9. 